\title{
New physics searches with EW penguins and radiative $B$ decays at $\mathrm{LHCb}$
}

\author{
Carla Marin Benito*广 \\ Universitat de Barcelona \\ carla.marinecern.ch
}

Rare $b \rightarrow s(\gamma, \ell \ell)$ decays are flavour changing neutral current processes that are forbidden at the lowest perturbative order in the Standard Model (SM). As a consequence, new particles in SM extensions can significantly affect the branching fractions of these decays and give rise to new sources of CP-violation. The LHCb experiment is ideally suited for the analysis of rare decays due to the large cross-section for $b \bar{b}$ production at the LHC, as well as its high trigger efficiency and excellent tracking, vertex reconstruction and particle identification capabilities. Recent results from the $\mathrm{LHCb}$ experiment in the area of semileptonic and radiative $b \rightarrow s$ transitions are presented and their interpretation is discussed.

The European Physical Society Conference on High Energy Physics

5-12 July, 2017

Venice

* Speaker.

${ }^{\dagger}$ On behalf of the LHCb Collaboration. 


\section{Introduction} tions entering the loop.

Rare decays of b-hadrons are Flavour Changing Neutral Currents (FCNC) that are forbidden at tree-level in the Standard Model (SM). Consequently they constitute an ideal place to search for New Physics (NP) effects since the decay properties are very sensitive to potential new contribu-

A model-independent description of rare decays is provided by the Operator Product Expansion [1], in which the effective Hamiltonian is decomposed as:

$$
H_{e f f} \propto \sum_{i}\left(C_{i}^{S M}+C_{i}^{N P}\right) \cdot O_{i}
$$

where $C_{i}$ and $O_{i}$ are the Wilson coefficients and operators representing the different interaction terms. The values of the Wilson coefficients can be extracted from global fits to the data. Any deviation from the SM predictions would be a clear sign of NP.

\section{Electroweak b decays}

Rare electroweak decays of b-hadrons provide a rich variety of observables to test the presence of NP in the penguin loop. Measurements of differential branching fractions $(\mathscr{B})$ in several $b \rightarrow$ $\mathrm{s} \ell^{+} \ell^{-}$decays by $\mathrm{LHCb}[2,3,4,5]$ show a systematic trend at low $q^{2}$ towards lower values with respect the SM prediction, as can be seen in Figure 1.
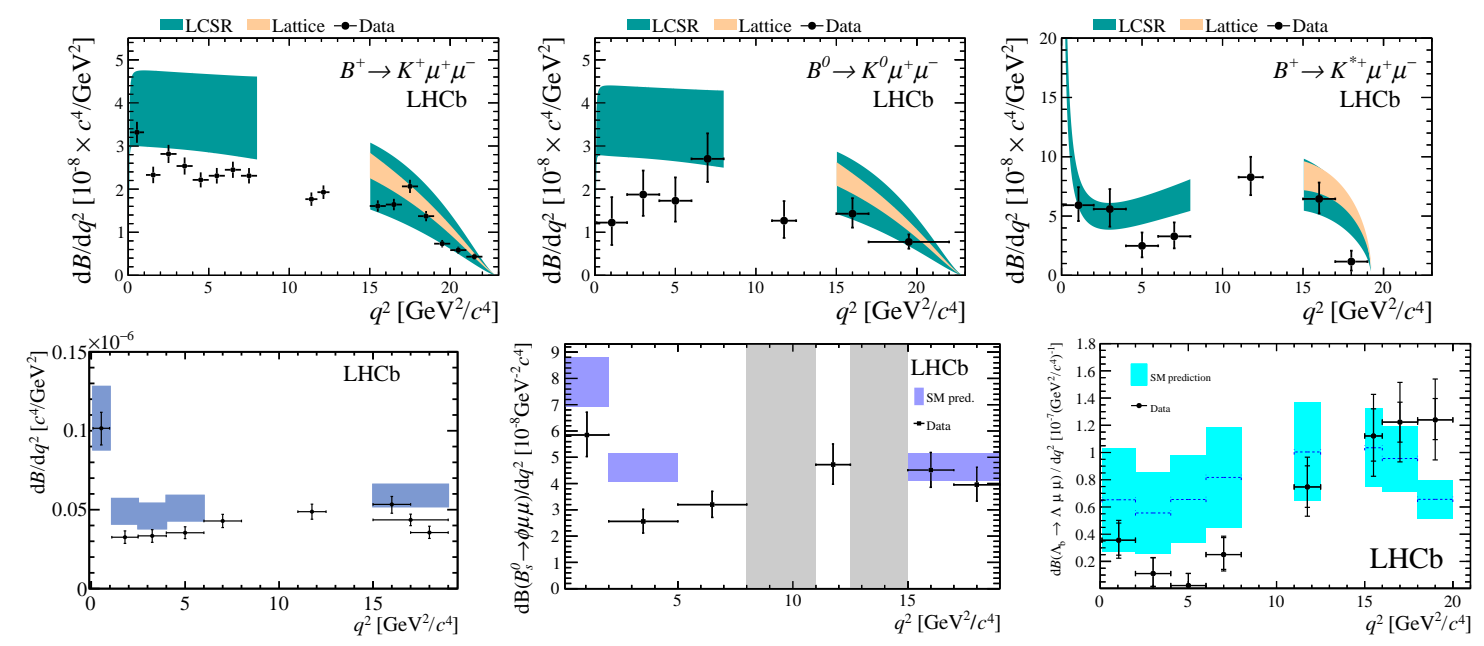

Figure 1: Differential branching fractions for (from top-left to bottom-right) $B^{+} \rightarrow \mathrm{K}^{+} \mu^{+} \mu^{-}, B^{0} \rightarrow$ $\mathrm{K}^{0} \mu^{+} \mu^{-}, B^{+} \rightarrow \mathrm{K}^{*+} \mu^{+} \mu^{-}, B^{0} \rightarrow \mathrm{K}^{* 0} \mu^{+} \mu^{-}, B_{\mathrm{s}}^{0} \rightarrow \phi \mu^{+} \mu^{-}$and $\Lambda_{\mathrm{b}}^{0} \rightarrow \Lambda \mu^{+} \mu^{-}$decays.

It should be noticed that this trend has not been observed in $\mathrm{b} \rightarrow \mathrm{d} \ell^{+} \ell^{-}$transitions [6], although the precision in these modes is lower so the situation is still inconclusive.

On top of updating these measurements with larger statistics it is important to explore other related modes to confirm the pattern. LHCb has recently observed for the first time the rare baryonic transitions $\Lambda_{\mathrm{b}}^{0} \rightarrow \mathrm{p} \pi^{-} \mu^{+} \mu^{-}$[7] and $\Lambda_{\mathrm{b}}^{0} \rightarrow \mathrm{pK}^{-} \mu^{+} \mu^{-}$[8]. The $\mathscr{B}$ of $\Lambda_{\mathrm{b}}^{0} \rightarrow \mathrm{p} \pi^{-} \mu^{+} \mu^{-}$is measured relative to $\Lambda_{\mathrm{b}}^{0} \rightarrow \mathrm{p} \pi^{-} \mathrm{J} / \psi$ and found to be:

$$
\mathscr{B}\left(\Lambda_{\mathrm{b}}^{0} \rightarrow \mathrm{p} \pi^{-} \mu^{+} \mu^{-}\right)=\left(6.9 \pm 1.9 \pm 1.1_{-1.0}^{+1.3}\right) \times 10^{-8} .
$$


This constitutes the first observation of a baryonic $b \rightarrow d$ transition.

For the $\Lambda_{\mathrm{b}}^{0} \rightarrow \mathrm{pK}^{-} \mu^{+} \mu^{-}$decay, Charge-Parity (CP) observables have been studied. In particular, the difference between the direct CP asymmetry in $\Lambda_{\mathrm{b}}^{0} \rightarrow \mathrm{pK}^{-} \mu^{+} \mu^{-}$and $\Lambda_{\mathrm{b}}^{0} \rightarrow \mathrm{pK}^{-} \mathrm{J} / \psi$, for which no asymmetry is expected, has been measured to be:

$$
\Delta A_{C P}=A_{C P}\left(\Lambda_{\mathrm{b}}^{0} \rightarrow \mathrm{pK}^{-} \mu^{+} \mu^{-}\right)-A_{C P}\left(\Lambda_{\mathrm{b}}^{0} \rightarrow \mathrm{pK}^{-} \mathrm{J} / \psi\right)=(-3.5 \pm 5.0 \pm 0.2) \times 10^{-2} .
$$

A complementary time-odd observable is constructed based on asymmetries in the angle between the $\mu^{+} \mu^{-}$and $\mathrm{pK}^{-}$decay planes, which has been found to be:

$$
a_{C P}^{\hat{T}-o d d}=(1.2 \pm 5.0 \pm 0.7) \times 10^{-2} .
$$

\section{Both observables are compatible with no CP violation.}

The invariant mass distributions of $\Lambda_{\mathrm{b}}^{0} \rightarrow \mathrm{p} \pi^{-} \mu^{+} \mu^{-}$and $\Lambda_{\mathrm{b}}^{0} \rightarrow \mathrm{pK}^{-} \mu^{+} \mu^{-}$candidates, split in $\mathrm{CP}$ eigenstates for the latter, are shown in Figure 2.
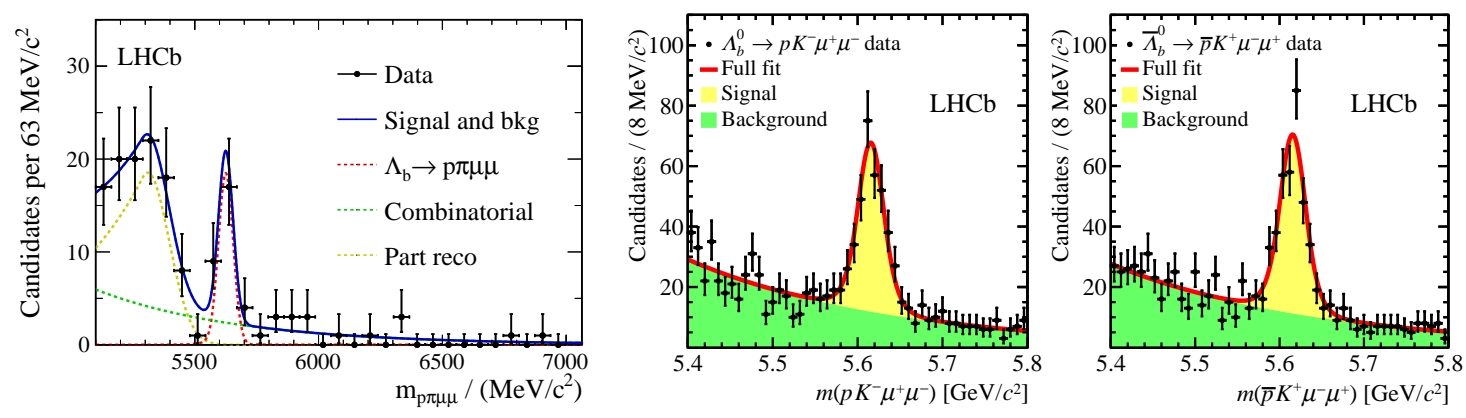

Figure 2: Invariant mass distribution of $\Lambda_{\mathrm{b}}^{0} \rightarrow \mathrm{p} \pi^{-} \mu^{+} \mu^{-}$(left) and $\Lambda_{\mathrm{b}}^{0} \rightarrow \mathrm{pK} \mathrm{K}^{-} \mu^{+} \mu^{-}$(right) candidates.

Angular observables in $\mathrm{b} \rightarrow \mathrm{s} \ell^{+} \ell^{-}$transitions are also very sensitive to NP, in particular the theoretically clean $P_{i}^{\prime}$ introduced in Ref. [9] that are free from form factor uncertainties at leading order. LHCb reported a deviation from the SM predictions in the differential distribution of $P_{5}^{\prime}$ in he $B^{0} \rightarrow \mathrm{K}^{* 0} \mu^{+} \mu^{-}$decay using the full Run 1 data sample [10]. More recently, Belle [11] and Atlas [12] have reported similar results, while CMS [13] observes a distribution compatible with the SM expectations and the LHCb measurement. Angular analyses of other decay modes are in greement with the SM predictions but they are not using such theoretically clean observables.

Global fits $[14,15,16,17,18]$ including both angular and $\mathscr{B}$ measurements together with recent tests of Lepton Flavour Universality (LFU) in $b \rightarrow s \ell^{+} \ell^{-}$decays $[19,20]$ point to additional NP contributions to the $C_{9}$ Wilson coefficient to explain the observed anomalies. However other authors suggest that a subset of the anomalies could be explained by hadronic effects [21, 22].

To study possible contributions from the charm-loop, a measurement of the phase difference between the short- and long-distance contributions to the decay $B^{+} \rightarrow \mathrm{K}^{+} \mu^{+} \mu^{-}$has been performed using LHCb Run 1 data corresponding to an integrated luminosity of $3 \mathrm{fb}^{-1}$ [23]. The long-distance contribution to the $B^{+} \rightarrow \mathrm{K}^{+} \mu^{+} \mu^{-}$decay is modelled as a sum of relativistic BreitWigner amplitudes representing the $\rho, \omega, \phi, \mathrm{J} / \psi, \psi(2 S), \psi(3770), \psi(4040), \psi(4160)$ and $\psi(4415)$ resonances decaying to muon pairs, each with their own magnitude and phase. The measured 
phases of the $\mathrm{J} / \psi$ and $\psi(2 S)$ resonances are such that the interference with the short-distance component in the dimuon mass region far from their pole masses is small. The full fit to the dimuon spectrum is shown in Figure 3 left. The branching fraction of the short-distance component is computed by setting the amplitudes of the resonances to zero:

$$
\mathscr{B}\left(B^{+} \rightarrow \mathrm{K}^{+} \mu^{+} \mu^{-}\right)=(4.37 \pm 0.15 \pm 0.23) \times 10^{-7}
$$

which is compatible with the previous analysis [2]. In addition, a likelihood scan of the Wilson coefficients $C_{9}$ and $C_{10}$ is performed taking into account both short- and long-distance contributions, leading to a deviation of around $3 \sigma$ with respect the SM value, as shown in Figure 3 right.
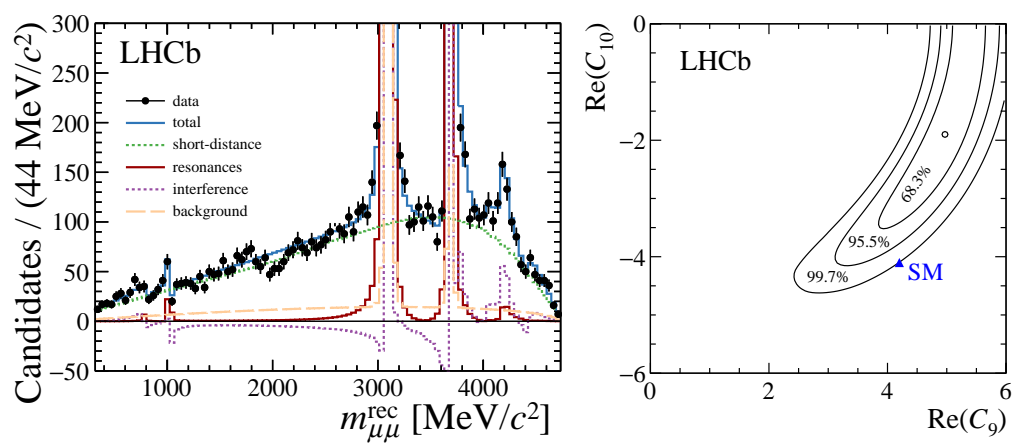

Figure 3: Dimuon invariant mass distribution of $B^{+} \rightarrow \mathrm{K}^{+} \mu^{+} \mu^{-}$(left). Likelihood scan of the Wilson coefficients $C_{9}$ and $C_{10}$ (right). The blue triangle shows the SM prediction.

\section{Radiative b decays}

Radiative $\mathrm{b} \rightarrow \mathrm{s} \gamma$ decays also provide interesting observables to search for NP effects. Both $\mathscr{B}$ and direct CP observables have been measured to be in good agreement with the SM [24]. However, there is still room for NP in the photon polarisation in such decays. The photon polarisation is predicted to be dominantly left-handed in the SM due to the absence of right-handed currents and is defined as:

$$
\alpha_{\gamma}^{S M}=\frac{P\left(\gamma_{L}\right)-P\left(\gamma_{R}\right)}{P\left(\gamma_{L}\right)+P\left(\gamma_{R}\right)}=1+\mathscr{O}\left(\frac{m_{s}}{m_{b}}\right) .
$$

New physics models could enhance the right-handed contribution up to 50\% [25] and thus a precise measurement of this observable is desirable.

A non-zero photon polarisation was observed for the first time in radiative b-decays in the mode $B^{+} \rightarrow \mathrm{K}^{+} \pi^{+} \pi^{-} \gamma$ using the full LHCb Run 1 dataset [26]. However, the exact value of the polarisation depends on the hadronic content and could not be determined.

The photon polarisation can also be accessed through the $B_{\mathrm{s}}^{0} \rightarrow \phi \gamma$ decay. In the case where the flavour of the initial meson is not known, the time-dependent decay rate can be written as:

$$
\Gamma_{B_{\mathrm{s}}^{0} \rightarrow \phi \gamma}(t) \propto e^{-\Gamma_{s} t}\left[\cosh \left(\Delta \Gamma_{s} t / 2\right)-A^{\Delta} \sinh \left(\Delta \Gamma_{s} t / 2\right)\right]
$$

where $A^{\Delta}$ is related to the photon polarisation. The SM prediction for this observable is $A^{\Delta}=$ $0.047_{-0.025}^{+0.029}$ [27]. LHCb has performed a time-dependent analysis of the decay rate of $B_{\mathrm{s}}^{0} \rightarrow \phi \gamma$ 
using the full Run 1 sample [28]. The $A^{\Delta}$ parameter is obtained from a fit to the background subtracted $B_{\mathrm{s}}^{0} \rightarrow \phi \gamma$ decay-time distribution shown in Figure 4 and is found to be:

$$
A^{\Delta}=-0.98_{-0.52}^{+0.46+0.23}
$$

which is compatible with the SM expectation at $2.6 \sigma$.

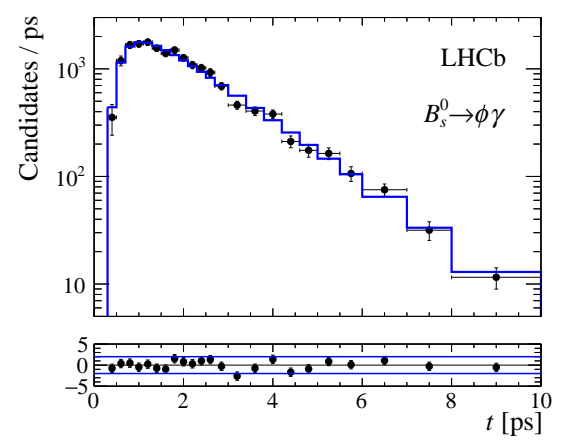

Figure 4: Fit to the $B_{\mathrm{s}}^{0} \rightarrow \phi \gamma$ decay-time distribution. Black dots represent the data points and the blue solid line the fitted distribution.

Baryonic $\mathrm{b} \rightarrow \mathrm{s} \gamma$ decays are also sensitive to the photon polarisation through angular analyses and are accessible at LHCb. For instance, for the decay $\Lambda_{\mathrm{b}}^{0} \rightarrow \Lambda \gamma$ the angular distributions of the final state particles are given by:

$$
\begin{aligned}
\frac{d \Gamma}{d \cos \theta_{\gamma}} & \propto 1-\alpha_{\gamma} P_{\Lambda_{\mathrm{b}}^{0}} \cos \theta_{\gamma} \\
\frac{d \Gamma}{d \cos \theta_{p}} & \propto 1-\alpha_{\gamma} \alpha_{p, 1 / 2} \cos \theta_{p}
\end{aligned}
$$

where $\alpha_{\gamma}$ is the photon polarisation, $P_{\Lambda_{\mathrm{b}}^{0}}=(0.06 \pm 0.07)$ [29] is the initial $\Lambda_{\mathrm{b}}^{0}$ polarisation and $\alpha_{p, 1 / 2}=(0.642 \pm 0.13)$ [24] is the weak parameter of the $\Lambda \rightarrow \mathrm{p} \pi^{-}$decay.

\section{Conclusions}

Rare FCNC b-decays provide clean observables to search for NP effects. Several tensions with respect to the SM predictions have been observed in $\mathscr{B}$ and angular observables of EW penguin transitions. Recent LFU tests point in the same direction. Updates of previous measurements and new analyses expanding the physics reach are ongoing to cross-check these observations.

For radiative b-decays the most promising observable to test the SM is the photon polarisation. A first measurement of this parameter by LHCb is reported. Analyses using more information and new decay modes are ongoing to obtain this observable with higher precision.

The exploitation of LHCb Run 2 data to study rare modes is ongoing and new results with higher precision are expected very soon.

\section{References}

[1] Kenneth G. Wilson. Non-lagrangian models of current algebra. Phys. Rev., 179:1499-1512, Mar 1969. 
[2] R. Aaij et al. Differential branching fractions and isospin asymmetries of $B \rightarrow K^{(*)} \mu^{+} \mu^{-}$decays. JHEP, 06:133, 2014.

[3] Roel Aaij et al. Measurements of the S-wave fraction in $B^{0} \rightarrow K^{+} \pi^{-} \mu^{+} \mu^{-}$decays and the $B^{0} \rightarrow K^{*}(892)^{0} \mu^{+} \mu^{-}$differential branching fraction. JHEP, 11:047, 2016.

[4] Roel Aaij et al. Angular analysis and differential branching fraction of the decay $B_{s}^{0} \rightarrow \phi \mu^{+} \mu^{-}$. JHEP, 09:179, 2015.

[5] Roel Aaij et al. Differential branching fraction and angular analysis of $\Lambda_{b}^{0} \rightarrow \Lambda \mu^{+} \mu^{-}$decays. JHEP, $06: 115,2015$.

[6] Roel Aaij et al. First measurement of the differential branching fraction and $C P$ asymmetry of the $B^{ \pm} \rightarrow \pi^{ \pm} \mu^{+} \mu^{-}$decay. JHEP, 10:034, 2015.

[7] Roel Aaij et al. Observation of the suppressed decay $\Lambda_{b}^{0} \rightarrow p \pi^{-} \mu^{+} \mu^{-}$. JHEP, 04:029, 2017.

[8] Roel Aaij et al. Observation of the decay $\Lambda_{b}^{0} \rightarrow p K^{-} \mu^{+} \mu^{-}$and a search for $C P$ violation. JHEP, 06:108, 2017

[9] Sébastien Descotes-Genon, Tobias Hurth, Joaquim Matias, and Javier Virto. Optimizing the basis of $B \rightarrow K^{*} \ell^{+} \ell^{-}$observables in the full kinematic range. Journal of High Energy Physics, 2013(5):137, May 2013.

[10] Roel Aaij et al. Angular analysis of the $B^{0} \rightarrow K^{* 0} \mu^{+} \mu^{-}$decay using $3 \mathrm{fb}^{-1}$ of integrated luminosity. JHEP, 02:104, 2016.

[11] A. Abdesselam et al. Angular analysis of $B^{0} \rightarrow K^{*}(892)^{0} \ell^{+} \ell^{-}$. In Proceedings, LHCSki 2016 - A First Discussion of 13 TeV Results: Obergurgl, Austria, April 10-15, 2016, 2016.

[12] Angular analysis of $B_{d}^{0} \rightarrow K^{*} \mu^{+} \mu^{-}$decays in $p p$ collisions at $\sqrt{s}=8 \mathrm{TeV}$ with the ATLAS detector. Technical Report ATLAS-CONF-2017-023, CERN, Geneva, Apr 2017.

[13] Measurement of the $P_{1}$ and $P_{5}^{\prime}$ angular parameters of the decay $\mathrm{B}^{0} \rightarrow \mathrm{K}^{* 0} \mu^{+} \mu^{-}$in proton-proton collisions at $\sqrt{s}=8 \mathrm{TeV}$. Technical Report CMS-PAS-BPH-15-008, CERN, Geneva, 2017.

[14] Bernat Capdevila, Andreas Crivellin, Sébastien Descotes-Genon, Joaquim Matias, and Javier Virto. Patterns of New Physics in $b \rightarrow s \ell^{+} \ell^{-}$transitions in the light of recent data. 2017.

[15] Wolfgang Altmannshofer, Peter Stangl, and David M. Straub. Interpreting hints for lepton flavor universality violation. Phys. Rev. D, 96:055008, Sep 2017.

[16] T. Hurth, F. Mahmoudi, D. Martinez Santos, and S. Neshatpour. On lepton non-universality in

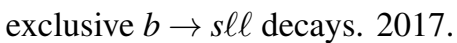

[17] Li-Sheng Geng, Benjamín Grinstein, Sebastian Jäger, Jorge Martin Camalich, Xiu-Lei Ren, and Rui-Xiang Shi. Towards the discovery of new physics with lepton-universality ratios of $b \rightarrow$ sll decays. 2017.

[18] Guido D'Amico, Marco Nardecchia, Paolo Panci, Francesco Sannino, Alessandro Strumia, Riccardo Torre, and Alfredo Urbano. Flavour anomalies after the $R_{K^{*}}$ measurement. JHEP, 09:010, 2017.

[19] R. Aaij et al. Test of lepton universality using $B^{+} \rightarrow K^{+} \ell^{+} \ell^{-}$decays. Phys. Rev. Lett., 113:151601, Oct 2014.

[20] R. Aaij et al. Test of lepton universality with $B^{0} \rightarrow K^{* 0} \ell^{+} \ell^{-}$decays. JHEP, 08:055, 2017.

[21] James Lyon and Roman Zwicky. Resonances gone topsy turvy - the charm of QCD or new physics in $b \rightarrow s \ell^{+} \ell^{-} ? 2014$. 
[22] Marco Ciuchini, Marco Fedele, Enrico Franco, Satoshi Mishima, Ayan Paul, Luca Silvestrini, and Mauro Valli. $B \rightarrow K^{*} \ell^{+} \ell^{-}$decays at large recoil in the Standard Model: a theoretical reappraisal. Journal of High Energy Physics, 2016(6):116, Jun 2016.

[23] Roel Aaij et al. Measurement of the phase difference between short- and long-distance amplitudes in the $B^{+} \rightarrow K^{+} \mu^{+} \mu^{-}$decay. Eur. Phys. J., C77(3):161, 2017.

[24] C. Patrignani et al. Review of particle physics. Chinese Phys. C, 40(100001), 2016.

[25] David Atwood, Michael Gronau, and Amarjit Soni. Mixing-induced $C P$ asymmetries in radiative $B$ decays in and beyond the standard model. Phys. Rev. Lett., 79:185-188, Jul 1997.

[26] Roel Aaij et al. First experimental study of photon polarization in radiative $B_{s}^{0}$ decays. Phys. Rev. Lett., 112(16):161801, 2014.

[27] Franz Muheim, Yuehong Xie, and Roman Zwicky. Exploiting the width difference in $B_{\mathrm{s}}^{0} \rightarrow \phi \gamma$. Physics Letters B, 664(3):174 - 179, 2008.

[28] Roel Aaij et al. First experimental study of photon polarization in radiative $B_{s}^{0}$ decays. Phys. Rev. Lett., 118(2):021801, 2017. [Addendum: Phys. Rev. Lett.118,no.10,109901(2017)].

[29] R Aaij et al. Measurements of the $\Lambda_{b}^{0} \rightarrow J / \psi \Lambda$ decay amplitudes and the $\Lambda_{b}^{0}$ polarisation in $p p$ collisions at $\sqrt{s}=7 \mathrm{TeV}$. Phys. Lett., B724:27-35, 2013. 